\title{
Minimal Realizations and Spectrum Generating Algebras
}

\author{
A. Joseph \\ Institut des Hautes Etudes Scientifiques, Bures-sur-Yvette, France
}

Received February 2, 1974

\begin{abstract}
The solution to the following problem is presented. Determine the least number of degrees of freedom for which a quantum mechanical system admits a given semisimple Lie algebra and construct the corresponding class of realizations. Such realizations are termed minimal realizations. It is shown that they can be obtained by a generalization of the inducing construction. Their physical importance is emphasized by showing that they possess most of the essential properties required of spectrum generating algebras.
\end{abstract}

\section{Introduction}

Spectrum generating algebras [1], (also called dynamical groups [2]) represent one of the most developed uses of Lie algebras in the description of elementary particle processes. Because they are required to possess an irreducible representation which exhausts the spectrum of a given system their construction involves the realization of a Lie algebra within a minimal number of degrees of freedom. By generalizing the notion of an induced representation we have obtained a new construction for such realizations which applies to all semisimple Lie algebras. Here we briefly outline the method, state the main theorems and present explicit formulae for all the classical simple Lie algebras and for $G_{2}$. For the special linear and the symplectic Lie algebras these reduce to certain well-known forms which have been used extensively in the context of spectrum generating algebras. These earlier results are thus set within a well-defined scheme which admits their extension to the orthogonal and the exceptional Lie algebras. Furthermore we show that many properties generally associated with spectrum generating algebras are also associated with minimal realizations. Moreover a minimal realization picks out a class of irreducible representations of the abstract Lie algebra which are naturally associated with canonical operators and for that reason are precisely the ones considered in quantum physics. Although the analysis is within the framework of quantum mechanics similar results can as usual be obtained in classical mechanics. 


\section{The Weyl Algebra}

The algebraic structure of quantum mechanics is based on the canonical commutation relations. For our present purposes these are most conveniently described as follows.

Let $\mathbb{C}$ denote the complex numbers and $n$ a positive integer. The Weyl algebra $\mathscr{A}_{n}(\mathbb{C})$, or simply $\mathscr{A}_{n}$, is the associative algebra over $\mathbb{C}$ with identity 1 defined on generators $q_{i}, p_{i}: i=1,2, \ldots n$, which satisfy

$$
\begin{aligned}
& q_{i} q_{j}-q_{j} q_{i}=p_{i} p_{j}-p_{j} p_{i}=0, \\
& q_{i} p_{j}-p_{j} q_{i}=\left\{\begin{array}{l}
0: i \neq j \\
1: i=j
\end{array}\right.
\end{aligned}
$$

Define the canonical action of $\mathscr{A}_{n}$ to be its action in the polynomial algebra $\mathbb{C}\left[q_{1}, q_{2}, \ldots q_{n}\right]$ in which $q_{i}$ becomes multiplication and $-p_{i}$ differentiation. $\mathscr{A}_{n}$ is isomorphic to the resulting algebra of differential operators over $\mathbb{C}\left[q_{1}, q_{2}, \ldots q_{n}\right] . \mathscr{A}_{n}$ admits $[3,4]$, pp. 163-167, a left quotient field $\mathscr{D}_{n}$ which may be viewed as the set $\left\{a^{-1} b: a, b \in \mathscr{A}_{n}\right\}$ with certain obvious identifications. $\mathscr{D}_{n}$ admits the subalgebra $\mathscr{B}_{n}=\left\{a^{-1} b: a\right.$ $\left.\in \mathbb{C}\left[q_{1}, q_{2}, \ldots q_{n}\right], b \in \mathscr{A}_{n}\right\}$ which is isomorphic to the algebra of differential operators over the field of rational functions $\mathbb{C}\left(q_{1}, q_{2}, \ldots q_{n}\right)$.

$\mathscr{A}_{n}$ (resp. $\mathscr{B}_{n}, \mathscr{D}_{n}$ ) may be considered as a Lie algebra (of infinite dimension) over $\mathbb{C}$ by defining the Lie bracket

$$
[a, b]=a b-b a,
$$

for all $a, b \in \mathscr{A}_{n}$ (resp. $\mathscr{B}_{n}, \mathscr{D}_{n}$ ). The Heisenberg algebra $\mathfrak{g}_{n}$ is the Lie algebra over $\mathbb{C}$ defined by (2.1) but in which 1 is replaced by a central element $z$. Then $\mathscr{A}_{n}$ is precisely the homomorphic image of the enveloping algebra $U \mathfrak{g}_{n}$ of $\mathfrak{g}_{n}$ with kernel $z-1$.

A Lie algebra gover $\mathbb{C}$ is said to admit a realization in $\mathscr{A}_{n}\left(\operatorname{resp} . \mathscr{B}_{n}, \mathscr{D}_{n}\right)$ if there exists an injective Lie algebra homomorphism $\varphi$ of $\mathfrak{g}$ into $\mathscr{A}_{n}$ (resp. $\mathscr{B}_{n}, \mathscr{D}_{n}$ ). Given dimg $<\infty, \mathfrak{g}$ admits [4], Chapter VI, a faithful finite dimensional representation $\varrho$. Let $\varrho_{i j}(x)$ denote the entries of the matrix $\varrho(x): x \in \mathfrak{g}$. Then

$$
\varphi(x)=\sum_{i j} \varrho_{i j}(x) q_{i} p_{j}
$$

defines a realization of $\mathfrak{g}$. Hence $g$ admits a realization in $\mathscr{A}_{n}$ for $n$ sufficiently large. A realization in $\mathscr{A}_{n}$ (resp. $\mathscr{B}_{n}, \mathscr{D}_{n}$ ) is called minimal if $n$ takes its smallest possible value, denoted by $n_{\mathscr{A}}(\mathfrak{g})\left[\right.$ resp. $\left.n_{\mathscr{B}}(\mathfrak{g}), n_{\mathscr{D}}(\mathfrak{g})\right]$. 
Obviously

$$
n_{\mathscr{A}}(\mathfrak{g}) \geqq n_{\mathscr{B}}(\mathfrak{g}) \geqq n_{\mathscr{D}}(\mathfrak{g})
$$

for all $\mathfrak{g}$. Furthermore [5]

Lemma 2.1. $n_{\mathscr{D}}\left(\mathfrak{g}_{n}\right)=n$ and a minimal realization $\varphi$ of $\mathfrak{g}_{n}$ in $\mathscr{D}_{n}$ satisfies $\varphi(z)=\alpha 1: \alpha \neq 0, \alpha \in \mathbb{C}$.

The weaker statement in which $\mathscr{B}$ replaces $\mathscr{D}$ is an easy consequence of [6], Theorem 1.1 and the relations (2.1). Observe that a minimal realization of $\mathfrak{g}_{n}$ defines an element of End $\mathscr{D}_{n}$, the semigroup of endomorphisms of $\mathscr{D}_{n}$.

Let $\varphi$ be a realization of $\mathfrak{g}$ in $\mathscr{A}_{n}$ (resp. $\mathscr{B}_{n}, \mathscr{D}_{n}$ ). Then $\varphi$ extends to an associative algebra homomorphism $\varphi^{*}$ of $U \mathfrak{g}$ into $\mathscr{A}_{n}$ (resp. $\mathscr{B}_{n}, \mathscr{D}_{n}$ ). $\varphi^{*}$ is almost never injective. Set $J_{\varphi}=\operatorname{ker} \varphi^{*}$. Recall that a two-sided ideal $J$ of $U \mathfrak{g}$ is said to be primitive [7] if it is the kernel of an irreducible representation of $\mathfrak{g}$. Let $D_{\varphi}(\mathfrak{g})$ denote the left quotient field of $\varphi^{*}(U \mathfrak{g})$.

Unless otherwise specified a Lie algebra is assumed to be over $\mathbb{C}$. Thus so $(n)$ denotes so $(n, \mathbb{C})$. A few remarks concern the real field $\mathbb{R}$. For this purpose, let $\mathscr{A}_{n}(\mathbb{R})$ denote the Weyl algebra over $\mathbb{R}$ and $\mathscr{D}_{n}(\mathbb{R})$ its left quotient field.

\section{Minimal Realizations of the Semisimple Lie Algebras}

Given $\mathfrak{g}$ semisimple it is well-known that $n_{\mathscr{B}}(\mathfrak{g}) \geqq$ rankg. (Actually $n_{\mathscr{D}}(\mathfrak{g}) \geqq$ rank $g$ has been shown [8], Theorem 4 , though by the implicit assumption of the first part of Lemma 2.1.) It turns out that this estimate is rather poor. (The failure of $n_{\mathscr{A}}(\mathfrak{g})=$ rank $\mathfrak{g}$ is noted in [9].) Here we show that $n_{\mathscr{D}}(\mathfrak{g})=n_{\mathscr{B}}(\mathfrak{g})$ and determine this integer. Unfortunately we have not been able to determine $n_{\mathscr{A}}(\mathfrak{g})$ completely though the uncertainty is small (see Table 1).

For $\mathfrak{g}$ semisimple

$$
n_{\mathscr{A}}(\mathfrak{g})=\sum_{i} n_{\mathscr{A}}\left(\mathfrak{g}_{i}\right)
$$

where $\mathfrak{g}_{i}$ denote the simple ideals of $\mathfrak{g}$. Corresponding formulae hold for $n_{\mathscr{B}}(\mathrm{g}), n_{\mathscr{D}}(\mathrm{g})$. Hence it suffices to consider $\mathrm{g}$ simple.

Assume $\mathfrak{g}$ simple. Then $\mathfrak{g}$ possesses a distinguished semisimple subalgebra $\mathfrak{g}^{\circ}$ which can be read off from the complete Dynkin diagram [10], pp. 198-200 for $\mathfrak{g}$. Indeed recall that the complete Dynkin diagram for $\mathfrak{g}$ is the Dynkin diagram for $\mathfrak{g}$ together with a specification of the Cartan inner product between the simple roots and the lowest root. The Dynkin 
diagram for $\mathfrak{g}^{\circ}$ is obtained by suppressing the simple roots not orthogonal to the lowest root. For example, given $\mathfrak{g}=\operatorname{sl}(n): n \geqq 3$, then $\mathfrak{g}^{\circ}=\operatorname{sl}(n-2)$.

Set

$$
k(\mathfrak{g})=\frac{1}{4}\left\{\left(\operatorname{dim} \mathfrak{g}-\operatorname{dim} \mathfrak{g}^{\circ}\right)-\left(\operatorname{rank} \mathfrak{g}-\operatorname{rank} \mathfrak{g}^{\circ}\right)+2\right\} .
$$

We showed in [11], Lemma 5.2 that

Lemma 3.1. A simple Lie algebra $\mathfrak{g}$ admits a Heisenberg subalgebra $\mathfrak{g}_{n}$ with $n=k(\mathfrak{g})-1$.

Now suppose $\mathfrak{g}$ admits a realization $\varphi$ in $\mathscr{D}_{n}: n=k(\mathfrak{g})-1$. By Lemmas 2.1 and 3.1 there exists $z \in \mathfrak{g}$ such that $\varphi(z)=\alpha 1$. Since $\varphi$ is injective $z \in C(\mathfrak{g})$, where $C(\mathfrak{g})$ denotes the centre of $\mathfrak{g}$. Yet $C(\mathfrak{g})=\{0\}$ for $\mathfrak{g}$ simple, hence

Lemma 3.2. $n_{\mathscr{D}}(\mathfrak{g}) \geqq k(\mathfrak{g})$.

However the interesting fact is that $n_{\mathscr{R}}(\mathfrak{g}) \leqq k(\mathfrak{g})$ which we prove constructively. By (2.2) there remains to determine $n_{\mathscr{A}}(\mathrm{g})$.

An upper bound on $n_{\mathscr{A}}(\mathfrak{g})$ can be obtained as follows. Given $\mathfrak{g}$ semisimple recall that it admits the canonical decomposition [12]

$$
\mathfrak{g}=\mathfrak{n}^{+} \oplus \mathfrak{h} \oplus \mathfrak{n}^{-}
$$

where $\mathfrak{h}$ is a Cartan subalgebra and $\mathfrak{n}^{ \pm}$are the nilpotent subalgebras spanned respectively by the positive and negative root eigenvectors. In addition the endomorphisms $\operatorname{ad}_{\mathfrak{g}} X: X \in \mathfrak{n}^{ \pm}$are nilpotent. A subalgebra of $\mathfrak{g}$ containing $\mathfrak{n}^{-} \oplus \mathfrak{h}$ is said to be parabolic. We remark that the Heisenberg subalgebra $\mathfrak{g}_{n}$ of Lemma 3.1 can be chosen such that $\mathfrak{g}_{n} C \mathfrak{n}^{+}$and the central element $z$ of $\mathfrak{g}_{\mathfrak{n}}$ is the highest root eigenvector of $\mathfrak{g}$.

Given any Lie algebra $\mathfrak{g}$ with subalgebra $\mathfrak{b}$ a representation $\varrho$ of $\mathfrak{g}$ induced from a one dimensional representation of $b$ can be realized [13] in a space of infinite order differential operators over a polynomial algebra of degree equal to $\operatorname{dim} \mathfrak{g}-\operatorname{dim} b$. (In the physics literature this is known as a non-linear [14,15] or an induced realization [16].) Now if $\mathfrak{b}$ possesses a complementary subalgebra $\mathfrak{p}$ such that every endomorphism $\operatorname{ad}_{\mathfrak{g}} X: X \in \mathfrak{p}$ is nilpotent, then the order of the differential operators corresponding to the elements of $g$ are bounded. In this case we obtain a realization of a homomorphic image $\theta(\mathfrak{g})$ of $\mathfrak{g}$ in $\mathscr{A}_{n}: n=\operatorname{dim} \mathfrak{g}$ $-\operatorname{dim} \mathfrak{b}$, which we shall call an induced realization.

Given $\mathfrak{g}$ semisimple the above condition is met if $\mathfrak{b}$ is parabolic. Furthermore given $\mathfrak{g}$ simple and $\mathfrak{b} \neq \mathfrak{g}$, then $\theta(\mathfrak{g})=\mathfrak{g}$. For $\mathfrak{g}$ simple, let $l(\mathfrak{g})$ be the infimum of $\operatorname{dim} \mathfrak{g}-\operatorname{dim} \mathfrak{b}$ as $\mathfrak{b}$ runs over the proper parabolic subalgebras of $\mathfrak{g}$. We have shown that

Lemma 3.3. $n_{\mathscr{A}}(\mathrm{g}) \leqq l(\mathrm{~g})$.

The integers $k(\mathfrak{g}), l(\mathfrak{g})$ are listed below for each simple Lie algebra. 
Table 1

\begin{tabular}{lllll}
\hline Cartan label & Classical Lie algebra & $k(\mathrm{~g})$ & $l(\mathrm{~g})$ & Remarks \\
\hline$A_{n}$ & $\operatorname{sl}(n+1)$ & $n$ & $n$ & $n \geqq 1$ \\
$B_{n}$ & $\operatorname{so}(2 n+1)$ & $2 n-2$ & $2 n-1$ & $n \geqq 3$ \\
$C_{n}$ & $\operatorname{sp}(2 n)$ & $n$ & $2 n-1$ & $n \geqq 2$ \\
$D_{n}$ & so $(2 n)$ & $2 n-3$ & $2 n-2$ & $n \geqq 4$ \\
$E_{6}$ & - & 11 & 16 & - \\
$E_{7}$ & - & 12 & 17 & - \\
$E_{8}$ & - & 34 & 67 & - \\
$F_{4}$ & - & 8 & 15 & - \\
$G_{2}$ & - & 3 & 5 & - \\
\hline
\end{tabular}

For $C_{n}$ we note in Section 7 that $n_{\mathscr{A}}(\mathrm{g})=n$. Hence $n_{\mathscr{A}}(\mathrm{g})$ is determined to within one for the classical Lie algebras. Our construction gives a realization of the orthogonal Lie algebras in one less degree of freedom than that obtained previously.

\section{The Construction of Minimal Realizations}

The construction of a minimal realization derives from Lemma 3.1. Thus we construct a realization of the Heisenberg algebra $\mathfrak{g}_{n}$ and attempt to extend this to a realization of $\mathfrak{g}$ itself. Since $\mathfrak{g}_{n} \mathrm{Cn}^{+}$the inducing procedure described in Section 3 can in principle be applied. However the requirement $\operatorname{dim} \mathfrak{p}=k(\mathfrak{g})$ on the complementary subspace $\mathfrak{p} \subset \mathfrak{g}_{n}$ excludes the realization of the whole of $g$ in this fashion (except for $A_{n}$ ). What we do is to obtain a realization of a certain parabolic subalgebra of $g$ and complete this to a realization of $\mathfrak{g}$ by implementing the action of the Weyl group in Aut $\mathscr{D}_{n}$. (See Section 9 for further details.) It is this latter step which pushes the realization outside $\mathscr{A}_{n}$. Indeed the realization always lies in the localization (see below) of $\mathscr{A}_{n}$ at $\varphi(z)$ where $z$ is the central element of $\mathfrak{g}_{n}$ being also the highest root eigenvector for $\mathfrak{g}$. Typically $\varphi(z)=q_{n}$. Define the localization $\mathscr{A}_{n}^{\prime}$ of $\mathscr{A}_{n}$ at $q_{n}$ through $\mathscr{A}_{n}^{\prime}=\left\{q_{n}^{-k} a: a \in \mathscr{A}_{n}: k=0,1,2 \ldots\right\}$. Obviously $\mathscr{A}_{n}^{\prime} \subset \mathscr{B}_{n}$. Identify two realizations $\varphi, \varphi^{\prime}$ in $\mathscr{D}_{n}$ if $\varphi^{\prime}=\theta \varphi$ for some $\theta \in$ Aut $\mathscr{D}_{n}$, the group of automorphisms of $\mathscr{D}_{n}$. It is shown in [5] that

Theorem 4.1. Each simple Lie algebra $\mathfrak{g}$ (excluding $A_{n}$ ) admits a unique minimal realization $\Phi$ in $\mathscr{D}_{n}$ with the following properties

(1) $n=k(g)$.

(2) $\Phi(\mathfrak{g}) \subset \mathscr{A}_{n}^{\prime}$.

(3) The Weyl group acts in $\Phi(\mathfrak{g})$ through Aut $\mathscr{D}_{n}$.

(4) $D_{\Phi}(g)=\mathscr{D}_{n}$.

(5) $J_{\Phi}$ is a primitive ideal of $U g$.

(6) Every realization $\varphi$ of $\mathfrak{g}$ in $\mathscr{D}_{n}$ takes the form $\varphi=\theta \Phi: \theta \in$ End $\mathscr{D}_{n}$. Furthermore $J_{\varphi}=J_{\Phi}$. 
Part (5) of the theorem implies $\varphi^{*}(C(U g)) \subset \mathbb{C} 1$, where $C(U g)$ denotes the centre of $U g$. Part (6) asserts the uniqueness of the primitive ideal. This is weaker than saying the corresponding irreducible representation is unique to within equivalence, which fails to hold (see Section 7). (This fact underlies the motivation for studying primitive ideals.)

The case of $A_{n}$ is described in the next section. In later sections we give the explicit form of the minimal realization for each simple Lie algebra (excluding $F_{4}, E_{6}, E_{7}, E_{8}$ though our method applies also to these cases).

$$
\text { 5. } A_{n}=\operatorname{sl}(n+1)
$$

Let $\left\{e_{i j}: i, j=1,2, \ldots n+1\right\}$ denote the canonical basis for $\mathrm{gl}(n+1)$ ( $e_{i j}$ is the matrix with one in the $i j^{\text {th }}$ entry and zeros elsewhere). The canonical decomposition (3.1) of $\mathrm{sl}(n+1)$ can be chosen such that $\left\{e_{i j}: i<j\right\}$ spans $n^{+}$. Then $\left\{e_{1 i}, e_{j n+1}: i=2,3, \ldots n+1, j=2,3, \ldots n\right\}$ spans the Heisenberg subalgebra $\mathfrak{g}_{n-1}$ of Lemma 3.1. The special nature of $\operatorname{sl}(n+1)$ derives from the fact that a maximal commutative subalgebra $\mathfrak{p}$ of $\mathfrak{g}_{n-1}$ given by $\mathfrak{p}=$ lin span $\left\{e_{1 i}: i=2,3 \ldots n+1\right\}$ is a complemented by a subalgebra $\mathfrak{b}$ of $\mathfrak{g}$. Consequently a representation of $\mathfrak{g}$ induced from a one-dimensional representation of $\mathfrak{b}$ determines a minimal realization of $\mathfrak{g}$ in $\mathscr{A}_{n}$. Furthermore, since the derived algebra $\mathfrak{b}^{\prime}$ of $\mathfrak{b}$ has codimension one, $\mathfrak{b}$ admits a one-parameter family of onedimensional representations. Hence we obtain a one parameter family of induced realizations $\varphi_{\alpha}: \alpha \in \mathbb{C}$, inequivalent $\bmod$ End $\mathscr{D}_{n}$. These are described below.

Let $\psi_{\alpha}: \alpha \in \mathbb{C}$ denote the linear map of $\operatorname{sl}(n+1)$ into $\mathscr{A}_{n}$ given by

$$
\begin{gathered}
\psi_{\alpha}\left(e_{1 i}\right)=q_{i-1}: i=2,3, \ldots n+1 \\
\psi_{\alpha}\left(e_{11}-e_{n+1 n+1}\right)=\alpha_{1} \\
\psi_{\alpha}(x)=0, \text { for all } x \in \mathfrak{b}^{\prime} .
\end{gathered}
$$

Set $A_{i}=\operatorname{ad} e_{1 i}, A=\sum_{i} p_{i} A_{i}$. Then $\varphi_{\alpha}$ is given by

$$
\varphi_{\alpha}(x)=: \psi_{\alpha}((\exp A) x):
$$

for all $x \in \operatorname{sl}(n+1)$. In (5.2) the dots denote normal ordering. That is the $p_{i}$ are taken outside $\psi_{\alpha}$ and are placed to the right. The relative simplicity of this formula derives from the fact that $\mathfrak{p}$ is commutative. We remark also that since $\mathfrak{p} \subset \mathfrak{n}^{+}$, it follows that $\varphi_{\alpha}(x) \in \mathscr{A}_{n}$ for each $X \in \mathfrak{g}$. When $\alpha=0, \varphi(\mathrm{g})$ is generated by $q_{i}, q_{i} p_{j},(q \cdot p) p_{i}: i, j=1,2 \ldots n$, where

$$
q \cdot p=\sum_{i=1}^{n} q_{i} p_{i}
$$


Under the canonical action of $\mathscr{A}_{n}, \varphi_{0}(g)$ acts in $\mathbb{C}\left[q_{1}, q_{2} \ldots q_{n}\right]$ and defines an irreducible representation of $\mathfrak{g}$. This holds for each $\alpha \in \mathbb{C}$. Indeed

Theorem 5.1. For each positive integer $n$, the simple Lie algebra $\mathfrak{g}=\mathrm{sl}(n+1)$ admits a one parameter family of minimal realizations $\varphi_{\alpha}: \alpha \in \mathbb{C}$ in $\mathscr{A}_{n}$ with the following properties

(1) The Weyl group acts in $\varphi_{\alpha}(\mathfrak{g})$ through Aut $\mathscr{D}_{n}$.

(2) $D_{\varphi_{\alpha}}(\mathfrak{g})=\mathscr{D}_{n}$, for each $\alpha \in \mathbb{C}$.

(3) $J_{\varphi_{\alpha}}: \alpha \in \mathbb{C}$ are distinct primitive ideals of $U \mathrm{~g}$. In particular the realizations $\varphi_{\alpha}$ are inequivalent $\bmod$ End $\mathscr{D}_{n}$.

(4) Every realization $\varphi^{\prime}$ of $\mathfrak{g}$ in $\mathscr{D}_{n}$ takes the form $\varphi^{\prime}=\theta \varphi_{\alpha}: \theta \in$ End $\mathscr{D}_{n}$, $\alpha \in \mathbb{C}$ and $J_{\varphi^{\prime}}=J_{\varphi_{\alpha}}$.

The case $n=3$ has been of physical interest. Recall the isomorphism $\operatorname{sl}(4, \mathbb{C})=\operatorname{so}(6, \mathbb{C})$. Real multiples of $q_{i}, q_{i} p_{j},(q \cdot p) p_{i}: i, j=1,2,3 ;$ generate the non-compact real form so $(4,2)$. This can be considered as a spectrum generating algebra of the hydrogen atom in momentum space $[1,17]$.

The statement corresponding to Theorem 5.1.4 with $\mathscr{A}_{n}$ replacing $\mathscr{D}_{n}$ is false. (See [18] and Section 7.)

\section{An Endomorphism of $\mathscr{D}_{n}$}

Define $\Theta \in$ End $\mathscr{D}_{n}$ through

$$
\begin{aligned}
& \Theta\left(q_{i}\right)=q_{n} q_{i}: i=1,2, \ldots n, \\
& \Theta\left(p_{i}\right)=q_{n}^{-1} p_{i}: i=1,2, \ldots n-1, \\
& \Theta\left(p_{n}\right)=1 / 2 q_{n}^{-2}\left\{q_{n} p_{n}-\sum_{1=1}^{n-1} q_{i} p_{i}\right\} .
\end{aligned}
$$

The presentation of the minimal realizations for the remaining simple Lie algebras is greatly facilitated by the use of $\Theta$. That is we replace the realization $\Phi$ of Theorem 4.1 by $\Psi=\Theta \Phi$. It should be noted that $\Psi$ does not share all the properties of $\Phi$. In any case $\Phi$ can be easily recovered from $\Psi$. A further advantage of $\Psi$ is that the canonical action of $\mathscr{A}_{n}$ leads quickly to an irreducible representation associated with $J_{\Phi}$.

$$
\text { 7. } C_{n}=\operatorname{sp}(2 n)
$$

By an accident $\Psi(\mathfrak{g}) \subset \mathscr{A}_{n}$ for $\mathfrak{g}=\operatorname{sp}(2 n)$, whereas this fails for $\Phi$. Furthermore $\Psi(\mathrm{g})$ is generated by the quadratic elements of $\mathscr{A}_{n}$ namely $q_{i} q_{j}, p_{i} p_{j},\left(q_{i} p_{j}+p_{j} q_{i}\right): i, j=1,2, \ldots n$. This shows that $n_{\mathscr{A}}(\mathfrak{g})=n_{\mathscr{B}}(\mathfrak{g}): \mathfrak{g}$ $=\operatorname{sp}(2 n)$. This realization of $\operatorname{sp}(2 n)$ is of course well-known [1]. It provides 
the spectrum generating algebra of the harmonic oscillator in $n$ dimensions, the case $n=3$ being of the most physical importance $[19,20]$.

Under the canonical action of $\mathscr{A}_{n}, \Psi(\mathrm{g})$ acts in $\mathbb{C}\left[q_{1}, q_{2}, \ldots q_{n}\right]$ and defines a reducible representation of $\mathfrak{g}$. This has two inequivalent irreducible components spanned by the odd and even polynomials. The corresponding primitive ideal is $J_{\Phi}$ in each case. Neither representation nor their direct sum can be considered as an induced representation of a proper subalgebra of $\mathfrak{g}$. This has been shown in [21], example 3, for the case $n=2$. In general it is a consequence of the fact that $\operatorname{sp}(2 n)$ admits no proper subalgebra of codimension less than or equal to $n$.

Taking $n=1$ in Section 5,7 we obtain two classes of realizations of sl(2) in $\mathscr{A}_{1}$ which are inequivalent mod End $\mathscr{A}_{1}$. These classes do not exhaust [18] all realizations of sl(2) in $\mathscr{A}_{1}$; but every realization admits a certain common spectral property [18], Theorem 2.6, with them. Does a corresponding result hold in $\mathscr{A}_{n}$ ? Finally note that the realization of sl(2) given above is equivalent to $\varphi_{-1 / 4} \bmod$ End $\mathscr{D}_{1}$.

\section{Description of the Simple Lie Algebras}

To define notation we recall some general properties of simple Lie algebras [22]. Let $\mathfrak{g}$ be a simple Lie algebra. With respect to (3.1) let $\Delta$ (resp. $\Delta^{+}$) denote the non-zero (resp. positive) roots. Let $\pi$ denote the set of simple roots, $\beta_{0}$ the highest root [10], pp. 165-6. Let (, ) denote the Cartan inner product. Let $E_{\alpha}: \alpha \in \Delta$ denote a root eigenvector with root $\alpha$. The $E_{\alpha}$ may be chosen such that

$$
\begin{aligned}
& {\left[E_{\alpha}, E_{\beta}\right]=0 \quad \alpha+\beta \notin \Delta, \alpha+\beta \neq 0} \\
& {\left[E_{\alpha}, E_{\beta}\right]=N_{\alpha \beta} E_{\alpha+\beta}: \alpha+\beta \in \Delta}
\end{aligned}
$$

where $N_{\alpha \beta}$ is real and

$$
N_{\alpha \beta}^{2}=\frac{r(1-s)}{2}(\alpha, \alpha)
$$

where $\beta+t \alpha: s \leqq t \leqq r$ is the $\alpha$-chain containing $\beta$.

Set

$$
H_{\alpha}=\left[E_{\alpha}, E_{-\alpha}\right] .
$$

The map $\alpha \rightarrow H_{\alpha}$ is linear, $\left\{H_{\alpha}: \alpha \in \Delta\right\}$ spans $\mathfrak{h}$ and $\left\{E_{\alpha}, H_{\alpha}: \alpha \in \Delta\right\}$ spans $\mathfrak{g}$. Equations (8.1)-(8.3) and

$$
\left[H, E_{\alpha}\right]=\alpha(H) E_{\alpha}: H \in \mathfrak{h}, \alpha \in \Delta
$$


define the commutation relations in $\mathfrak{g}$ (up to sign) given $\alpha\left(H_{\alpha}\right)=(\alpha, \alpha)$. (The signs can be obtained by explicit construction of the Lie algebras [4], Chapter IV, Section 6 or from [23].)

Let $\omega_{\alpha}$ denote the reflection in $\mathfrak{h}^{*}$ defined by the plane normal to $\alpha$. The group generated by $\left\{\omega_{\alpha}: \alpha \in \Delta\right\}$ is called the Weyl group $W$. $W$ leaves $\Delta$ stable and acts in $\mathfrak{h}$ through the map $\alpha \rightarrow H_{\alpha}$. It is generated [4], p. 241, by $\left\{\omega_{\alpha_{i}}: \alpha_{i} \in \pi\right\}$. It should be noted that there is no connection between the Weyl group and the Weyl algebra.

\section{9. $G_{2}$}

$G_{2}$ is the lowest rank Lie algebra for which our construction gives a novel realization. Set $\pi=\left\{\alpha_{1}, \alpha_{2}\right\}$, then [10], p. 276

$$
\Delta^{+}=\left\{\alpha_{1}, \alpha_{2}, \alpha_{1}+\alpha_{2}, 2 \alpha_{1}+\alpha_{2}, 3 \alpha_{1}+\alpha_{2}, 3 \alpha_{1}+2 \alpha_{2}\right\} \text {. }
$$

Hence $\beta_{0}=3 \alpha_{1}+2 \alpha_{2}$. Since $\left(\alpha_{1}, \beta_{0}\right)=0,\left(\alpha_{2}, \beta_{0}\right) \neq 0$, it follows that $\mathfrak{g}^{\circ}=\operatorname{sl}(2)$ with simple root $\alpha_{1}$ (cf. Section 2). Define

$$
I=\frac{1}{2}\left(E_{\alpha_{1}} E_{-\alpha_{1}}+E_{-\alpha_{1}} E_{\alpha_{1}}\right)-\frac{9}{4} H_{\alpha_{1}}^{2}-1 / 6 .
$$

Apart from the constant, $I$ is the quadratic Casimir invariant for $\mathfrak{g}^{\circ}$. We give the minimal realization $\Psi$ of $G_{2}$ in $\mathscr{A}_{3}^{\prime}$ below. For convenience we omit $\Psi$, that is we identify $\Psi\left(E_{\alpha}\right)$ with $E_{\alpha}$.

$$
\begin{aligned}
& E_{\alpha_{1}}=q_{2} p_{1}+\frac{1}{\sqrt{3}} p_{2}^{2}, \quad E_{\alpha_{2}}=q_{1} q_{3}, \\
& E_{\alpha_{1}+\alpha_{2}}=q_{2} q_{3}, \quad E_{2 \alpha_{1}+\alpha_{2}}=q_{3} p_{2}, \\
& E_{3 \alpha_{1}+\alpha_{2}}=q_{3} p_{1}, \quad E_{3 \alpha_{1}+2 \alpha_{2}}=q_{3}^{2} \text {, } \\
& E_{-\alpha_{1}}=-q_{1} p_{2}+\frac{1}{\sqrt{3}} q_{2}^{2}, \quad H_{\alpha_{1}}=q_{1} p_{1}+\frac{1}{3} q_{2} p_{2} \text {, } \\
& I=-\frac{1}{4}\left(q_{1} p_{1}+q_{2} p_{2}-1\right)^{2}+1 / 3\left(q_{2} p_{2}-1 / 2\right)^{2}+\frac{1}{3 \sqrt{3}}\left(q_{2}^{3} p_{1}-q_{1} p_{2}^{3}\right) \\
& E_{-\alpha_{2}}=\left[q_{3}^{-1} p_{1}, J\right], \quad E_{-\alpha_{1}-\alpha_{2}}=\left[q_{3}^{-1} p_{2}, J\right], \\
& E_{-2 \alpha_{1}-\alpha_{2}}=\left[q_{3}^{-1} q_{2}, J\right], \quad E_{-3 \alpha_{1}-\alpha_{2}}=\left[q_{3}^{-1} q_{1}, J\right], \\
& E_{-3 \alpha_{1}-2 \alpha_{2}}=q_{3}^{-2}\left(I+\frac{1}{4}\left(q_{3} p_{3}+3\right)^{2}+5 / 36\right), H_{3 \alpha_{1}+2 \alpha_{2}}=q_{3} p_{3}+2,
\end{aligned}
$$

where

$$
J=I-1 / 4\left(q_{3} p_{3}+2\right)^{2} .
$$


The commutation relations (8.1)-(8.4) may be verified by explicit computation. The Heisenberg subalgebra $g_{2}$ of Lemma 3.1 is spanned by $\left\{E_{\alpha}: \alpha \in \Delta^{+} ; \alpha \neq \alpha_{1}\right\}$. The Weyl group acts through Aut $\Theta \mathscr{D}_{3}$. This action may be determined by examining the image of $\mathfrak{g}_{2} \oplus \mathbb{C} H_{\beta_{0}}$ which generates a $\Theta \mathscr{D}_{3}$. For example $\omega_{\alpha_{1}}$ is given by

$$
\begin{gathered}
\omega_{\alpha_{1}}\left(q_{i}\right)=p_{i}, \quad \omega_{\alpha_{1}}\left(p_{i}\right)=-q_{i}: i=1,2, \\
\omega_{\alpha_{1}}\left(q_{3}\right)=q_{3}, \quad \omega_{\alpha_{1}}\left(p_{3}\right)=p_{3} .
\end{gathered}
$$

Extend the canonical action of $\mathscr{A}_{3}$ in $\mathbb{C}\left[q_{1}, q_{2}, q_{3}\right]$ to an action of $\mathscr{A}_{3}^{\prime}$ in $\mathbb{C}\left(q_{1}, q_{2}, q_{3}\right)$. Then $G_{2}$ acts in $\mathbb{C}\left(q_{1}, q_{2}, q_{3}\right)$. The vector space generated by the constant function defines an irreducible representation of $G_{2}$. This representation is not an induced representation of a proper subalgebra. On the other hand the restriction of $\Phi$ to the parabolic subalgebra $\mathfrak{f}=\mathfrak{n}^{+} \oplus \mathfrak{h} \oplus \mathbb{C} E_{-\alpha_{2}}$ of $\mathfrak{g}$ is an induced realization from its subalgebra $\mathfrak{b}=\mathbb{C} E_{2 \alpha_{1}+\alpha_{2}} \oplus \mathbb{C} E_{3 \alpha_{1}+\alpha_{2}} \oplus \mathbb{C} E_{\alpha_{1}} \oplus \mathfrak{h} \oplus \mathbb{C} E_{-\alpha_{2}}$. The complement $\mathfrak{p}=\mathbb{C} E_{\alpha_{2}} \oplus \mathbb{C} E_{\alpha_{1}+\alpha_{2}} \oplus \mathbb{C} E_{3 \alpha_{1}+2 \alpha_{2}}$ of $\mathfrak{b}$ in $\mathfrak{f}$ is a maximal commutative subalgebra of $\mathfrak{g}_{2}$. The point is that $\mathfrak{p}$ is not complemented in $\mathfrak{g}$ by a subalgebra and for this reason we require a generalization of the inducing procedure to obtain a minimal realization.

We draw attention to a characteristic feature of minimal realizations illustrated by (9.5). That is the Casimir invariant of a subalgebra, $I$ in this case, appears in the expression for certain elements of the complementary subspace. This result is reminiscent of a procedure (see, for example [24], Eq. (80)) whereby a Lie algebra realization is obtained from that of a subalgebra by a similar use of the invariants of the subalgebra. Yet this construction is not entirely general for there is no a priori reason why the resulting expressions should close on a Lie algebra and certainly no guarantee that the realization obtained be minimal. Indeed this is generally not the case (see Section 11). Essentially these invariants occur because $J_{\Phi}$ is very large and because elements of the complementary subspace must transform correctly under the subalgebra. Yet for physical considerations this feature is important since $I$, or a function of $I$, may be regarded as the Hamiltonian for a system whose symmetry is described by $\mathfrak{g}^{\circ}$ and whose spectrum generating algebra is $\mathfrak{g}$.

$$
\text { 10. } B_{n}=\operatorname{so}(2 n+1): n \geqq 3
$$

According to Table $1, B_{n}$ admits a minimal realization in $\mathscr{B}_{2 n-2}$. Set $\pi=\left\{\alpha_{i}: i=1,2, \ldots n\right\}$. We may write $\alpha_{i}=w_{i}-w_{i+1}: i=1,2, \ldots n-1$; $\alpha_{n}=w_{n}$. Then [4], p. 138, [10], p. 254,

$$
\Delta^{+}=\left\{w_{i}, w_{i}-w_{j}, w_{i}+w_{j}: i<j ; i, j=1,2, \ldots n\right\} .
$$


In addition

$$
\left(w_{i}, w_{j}\right)= \begin{cases}0 & i \neq j, \\ 1 & i=j .\end{cases}
$$

We have $\beta_{0}=w_{1}+w_{2}$. Since $\left(\alpha_{i}, \beta_{0}\right)=0$ :

$i=1,3,4 \ldots n$, it follows that $\mathfrak{g}^{\circ}=\operatorname{sl}(2) \oplus \operatorname{so}(2 n-3)$. In this the first component is defined through $\pi=\left\{\alpha_{1}\right\}$, the second through $\pi$ $=\left\{\alpha_{3}, \alpha_{4}, \ldots \alpha_{n}\right\}$. The following equations define $\Psi$ for these two subalgebras. Set

$$
\begin{gathered}
a_{i}=q_{2 i-4}, b_{i}=p_{2 i-4}, c_{i}=q_{2 i-5}, d_{i}=p_{2 i-5}: i=3,4, \ldots n, \\
e=q_{2 n-3}, f=p_{2 n-3}, g=q_{2 n-2}, h=p_{2 n-2} .
\end{gathered}
$$

Then the sl(2) factor is defined by

$$
\begin{gathered}
E_{w_{1}-w_{2}}=a \cdot d+1 / 2 f^{2}, \quad E_{-\left(w_{1}-w_{2}\right)}=-b \cdot c+1 / 2 e^{2}, \\
H_{w_{1}-w_{2}}=a \cdot b-c \cdot d-(e f-1 / 2),
\end{gathered}
$$

where the dot denotes summation over $i$. The so $(2 n-3)$ factor is defined by

$$
\begin{gathered}
E_{w_{j}+w_{k}}=b_{j} d_{k}-b_{k} d_{j}, \quad E_{-w_{j}-w_{k}}=a_{j} c_{k}-a_{k} c_{j}, \\
E_{w_{j}-w_{k}}=-\left(a_{k} b_{j}+c_{k} d_{j}\right), \quad E_{-\left(w_{j}-w_{k}\right)}=a_{j} b_{k}+c_{j} d_{k}, \\
E_{w_{j}}=-\left(e d_{j}+f b_{j}\right), \quad E_{-w_{j}}=f c_{j}-e a_{j}, \\
H_{w_{j}}=-\left(c_{j} d_{j}+a_{j} b_{j}-1 / 2\right),
\end{gathered}
$$

with $j<k: j, k=3,4, \ldots n$. Set

$$
I=\frac{1}{2}\left(E_{w_{1}-w_{2}} E_{-\left(w_{1}-w_{2}\right)}+E_{-\left(w_{1}-w_{2}\right)} E_{w_{1}-w_{2}}\right)-1 / 4 H_{w_{1}-w_{2}}^{2} .
$$

This is the quadratic Casimir invariant for the sl(2) factor. It is also the Casimir invariant for the so $(2 n-3)$ factor for this realization. The Heisenberg subalgebra $\mathfrak{g}_{2 n-2}$ is defined by

$$
\begin{gathered}
E_{w_{1}}=g f, \quad E_{w_{2}}=g e, \quad E_{w_{1}+w_{2}}=g^{2}, \\
E_{w_{1}-w_{i}}=g a_{i}, \quad E_{w_{2}-w_{\imath}}=g c_{i}, \\
E_{w_{1}+w_{2}}=g d_{i}, \quad E_{w_{2}+w_{i}}=g b_{i}
\end{gathered}
$$

$i=3,4, \ldots n$. Its counterpart in $n^{-}$together with $H_{\beta_{0}}$ is given by

$$
E_{-w_{1}-w_{2}}=g^{-2}\left(I+1 / 4(g h+7 / 2)^{2}-1 / 4\right), H_{w_{1}+w_{2}}=g h+5 / 2,(10.7)
$$

and commutation with (10.6), using (8.1) and (8.2). 
11. $D_{n}=\operatorname{so}(2 n): n \geqq 4$

According to Table $1, D_{n}$ admits a realization in $\mathscr{B}_{2 n-3}$. Set $\pi=\left\{\alpha_{i}: i=1,2, \ldots n\right\}$. We may write $\alpha_{i}=w_{i}-w_{i+1}: i=1,2, \ldots n-1$, $\alpha_{n}=w_{n-1}+w_{n}$. Then

$$
\Delta^{+}=\left\{w_{i}-w_{j}, w_{i}+w_{j}: i<j ; i, j=1,2, \ldots n\right\} .
$$

Comparison of (10.1) and (11.1) shows that $D_{n}$ is obtained from $B_{n}$ by suppressing the roots $w_{i}$. Correspondingly we obtain $\Psi$ from (10.3), (10.4), (10.6) by setting $e=f=0$ and adjusting certain numerical factors which arise from the commutation of $e$ and $f$. Thus we require

$$
\begin{gathered}
H_{w_{1}-w_{2}}=a \cdot b-c \cdot d \\
E_{-w_{1}-w_{2}}=g^{-2}\left(I+1 / 4(g h+3)^{2}-1 / 4\right), \quad H_{w_{1}+w_{2}}=g h+2
\end{gathered}
$$

instead of the expressions appearing in (10.3) and (10.7). This and the previous section give minimal realizations for $\operatorname{so}(n, \mathbb{C}): n \geqq 7$. For the low dimensional cases the following isomorphisms (over $\mathbb{C}$ ) should be noted.

$$
\text { so(3) }=\operatorname{sl}(2), \operatorname{so}(4)=\operatorname{sl}(2) \oplus \operatorname{sl}(2), \operatorname{so}(5)=\operatorname{sp}(4), \operatorname{so}(6)=\operatorname{sl}(4) \text {. }
$$

The subalgebras $\mathfrak{p}_{1}=$ lin $\operatorname{span}\left\{E_{w_{1}}, E_{w_{1}-w_{t}}, E_{w_{1}+w_{t}}: i=2,3, \ldots n\right\}, \mathfrak{p}_{2}$ $=$ lin $\operatorname{span}\left\{E_{w_{1}-w_{2}}, E_{w_{1}+w_{i}}: i=2,3, \ldots n\right\}$ are complementary to maximal parabolic subalgebras of so $(2 n+1)$ and so $(2 n)$ respectively. Moreover $\mathfrak{p}_{1}, \mathfrak{p}_{2}$ are commutative, so (5.2) applies to give a one-parameter family of induced realizations of so $(m+2): m \geqq 3$ in $\mathscr{A}_{m}$. This family of realizations is equivalent under a linear automorphism of $\mathscr{A}_{m}(\mathbb{C})$ (but not $\left.\mathscr{A}_{m}(\mathbb{R})\right)$ to that defined in the special case $m=3$ by [24], Eq. (10), (80). In this we remark that the familiar bilinear realization of so $(m)$ : namely that defined by generators $q_{i} p_{j}-q_{j} p_{i}: i, j=1,2, \ldots m$ is lifted by a twofold process to a realization of so $(m+2)$. The first step is the inverse stereographic projection [24], Eq. (10) taking so $(m)$ to so $(m+1)$, the second the expansion procedure [24], Eq. (80) taking so $(m+1)$ to so $(m+2)$. The additional generators can be chosen as $q_{i},(q \cdot p+\alpha) p_{i}$ $-1 / 2 q_{i} p^{2}: i=1,2, \ldots m ;(q \cdot p+\alpha): \alpha \in \mathbb{C}$ being the defining parameter. This realization of so $(m+2)$ can be considered as a spectrum generating algebra for the $m$ dimensional hydrogen atom in momentum space [24]. Considered as a realization in $\mathscr{B}_{m}$ it is not minimal; but this does not exclude the possibility of it being minimal in $\mathscr{A}_{m}$. In $\mathscr{A}_{m}(\mathbb{R})$ the corresponding realizations give the non-compact real form so $(m+1,1)$. 


\section{Casimir Invariants and Real Forms}

Let $C(U \mathfrak{g})$ denote the centre of $U \mathfrak{g}$. For $\mathfrak{g}$ semisimple it is a polynomial algebra generated by the Casimir invariants. For a minimal realization $\varphi$ in $\mathscr{D}_{n}$ we have $\varphi^{*}(C(U \mathfrak{g})) \subset \mathbb{C}$. This has the following consequence.

Lemma 12.1. Let $\mathfrak{g}$ be a compact semisimple Lie algebra over $\mathbb{R}$. Then $\mathrm{g}$ does not admit a realization in $\mathscr{D}_{n}(\mathbb{R})$ with $n=k(\mathrm{~g})$.

For example so $(5, \mathbb{R})$ cannot be realized in $\mathscr{D}_{2}(\mathbb{R})$. A similar statement is pointed out in [9]. The problem of determining minimal realizations in $\mathscr{D}_{n}(\mathbb{R})$ for all real forms is thus as yet unsolved.

The fact that it is always a non-compact real form which admits a realization in $\mathscr{D}_{n}(\mathbb{R}): n=k(\mathrm{~g})$ may be thought to be related to the fact that the corresponding representations are infinite dimensional whilst the unitary irreducible representations of compact groups are finite dimensional. This relationship is not quite direct, as noted in [25]. Dashen and Gell-Mann [26] stressed the use of non-compactness in spectrum generating algebras because of the infinite nature of the spectrum. We believe that the concept of a minimal realization serves this purpose just as well. Furthermore it gives a much more precise answer as to which representation to pick.

The statement $\varphi^{*}(C(U \mathfrak{g})) \subset \mathbb{C} 1$ for minimal realizations admits the following generalization.

Lemma 12.2. Let $\mathfrak{g}$ be semisimple, $\varphi$ a realization of $\mathfrak{g}$ in $\mathscr{B}_{n}$. Then $d(\mathfrak{g})+k(\mathfrak{g}) \leqq n$, where $d(\mathfrak{g})$ denotes the transcendence degree of $\varphi^{*}(C(U \mathfrak{g}))$ over $\mathbb{C}$.

We have been unable to prove a corresponding result for $\mathscr{D}_{n}$. The difficulty lies in extending [6], Theorem 11 to $\mathscr{D}_{n}$.

\section{References}

1. Ne'eman, Y.: Algebraic theory of particle physics, Chapter 10. New York: W. A. Benjamin 1967

2. Barut,A. O.: Application of the dynamical group theory to the structure of the hadrons. Lectures in theoretical physics (Ed. Barut, A. O., Brittin, W.E.), Vol. 10 B, pp. 377-426. New York: Gordon and Breach 1967

3. Gelfand,I.M., Kirillov, A. A.: Inst. Hautes Etudes Sci. Publ. Math. 31, 5 (1966)

4. Jacobson, N.: Lie algebras. New York: Interscience 1962

5. Joseph, A.: To appear

6. Joseph, A.: Ann. Inst. H. Poincaré 17, 325 (1972)

7. Dixmier,J.: Algèbres enveloppantes, Chapter 3. Paris: Gauthier-Villars, in press

8. Simoni, A., Zaccaria, F.: Nuovo Cimento 59 A, 280 (1969)

9. Mukunda, N.: J. Math. Phys. 8, 1069 (1967)

10. Bourbaki, N.: Groupes et algèbres de Lie, Chapter 4-6. Paris: Hermann 1968 
11. Joseph, A.: A generalization of the Gelfand-Kirillov conjecture. Tel-Aviv University Preprint 385 (1973)

12. Serre,J.-P.: Algèbres de Lie semi-simples complexes. Chapter VI, Theorem 4. New York: W. A. Benjamin 1966

13. Conze, N.: Bull. Math. Soc. France, in press

14. Coleman,S., Wess, J., Zumino, B.: Phys. Rev. 177, 2239 (1969)

15. Joseph, A., Solomon, A. I.: J. Math. Phys. 11, 748 (1970)

16. Ndili, F. N.: Lett. Nuovo Cimento 1, 13 (1971)

17. Kleinert, H.: Group dynamics of the hydrogen atom. Lectures in theoretical physics (Ed. Barut, A.O., Brittin,W.E.), Vol. 10B, pp. 437-446. New York: Gordon and Breach 1967

18. Joseph, A.: Proc. Camb. Phil. Soc., in press

19. Goshen, S., Lipkin, H.J.: Ann. Phys. 6, 301 (1959)

20. Lipkin, H.J.: Lie groups for pedestrians, p. 69. Amsterdam: North Holland Publ. Co. 1965

21. Conze, N., Dixmier, J.: Bull. Sci. Math., 2 série 96, 339 (1972)

22. Helgason, N.: Differential geometry and symmetric spaces. Chapter III, Sections 4, 5. New York: Academic Press 1962

23. Tits, J.: Inst. Hautes Etudes Sci. Publ. mathématiques 31, 21 (1966)

24. Bander, M., Itzykson, C.: Rev. Mod. Phys. 38, 330 (1966)

25. Joseph, A.: J. Math. Phys. 13, 351, Theorem 4.4 (1972)

26. Dashen, R., Gell-Mann, M.: Phys. Letters 17, 142 (1965)

Communicated by R. Haag

\author{
A. Joseph \\ Department of Physics \\ and Astronomy \\ Tel-Aviv University \\ Ramat Aviv, Israel
}

